\title{
Time, Interference, and Rate of Presentation in Short-Term Recognition Memory for Items ${ }^{1}$
}

\author{
WAyNe A. WrCKelGREN ${ }^{2}$ \\ Massachusetts Institute of Technology, Cambridge, Massachusetts 02139
}

\begin{abstract}
A list of 9 or 15 letters was presented at a rate of 1, 2, or 4 letters per second, followed by a test letter, followed by the subject's decision as to whether the test letter appeared in the previous list. The decay of trace strength was a simple exponential decay function of delay. However, the rate of decay was not constant for different rates of presentation, whether delay was measured in time or intervening items. Rather, time decay rate increased approximately linearly with presentation rate, but with a positive intercept, not a zero intercept as required by the hypothesis that delay is measured in intervening items. Decay rate for previous items appears to be greater during the time (less than $0.25 \mathrm{sec}$.) for acquisition of an item than during the time between acquisition of adjacent items.
\end{abstract}

This paper is concerned with the question of whether forgetting in short-term memory (STM) is primarily a function of temporal decay in the absence of rehearsal or of the interfering effects of subsequent items. One approach to this problem has been to vary the rate of presentation of a list of items to determine if more rapidly presented items are remembered better because they have had less time to decay, though the number of intervening items is held constant.

\section{Multiple Recall}

Almost all of the previous studies on this topic have used the method of "multiple recall" of all or many of the items in a list. This method has the advantage of efficiency of data collection, but it has the severc disadvantage that the retrieval process is too complicated to analyze precisely. Without an established, precise theory of retrieval, we are severely handicapped in separating acquisition and storage effects when they are confounded experimentally. Since varying the rate of presentation confounds temporal delay in storage with the time for acquisition of each item, it is not surprising

1 'This work was supported primarily by Grant, MH 08890-04, from the National Institute of Mental Health, U. S. Public Health Service. Further aid was received from a National Aeronautics and Space Administration Grant NsG 496, to Hans-Lukas Teuber. Susan Urban and Gail Nashner assisted in preparation and execution of the experiments.

${ }^{2}$ Now at L. of Oregon, Eugene, Ore. 97403. 
that studies of rate of presentation in STM using the method of multiple recall have obtained conflicting results.

Most studies have found that a slower rate of presentation facilitates performance in memory span tests (see reviews by Aaronson, 1967, and Posner, 1963). As Posner and Aaronson have pointed out, the facilitation of STM with decreased rate of presentation is presumably due to the increased time for perception, organization, and rehearsal of the list, which increases degree of learning (acquisition) in STM.

What is more significant is that some studies have actually found faster rates of presentation to facilitate short-term recall, despite the confounding factor of decreased time for acquisition (Conrad, 1957; Conrad and Hille, 1958; Mackworth, 1964; Posner, 1964). Slight support for temporal decay was also found by Schonfield and Donaldson (1966) in a study which varied the rate of presentation within a single list. Although the vast majority of studies have found faster presentation to be deleterious for short-term recall, the number of studies finding faster presentation to aid shortterm recall seems too great to pass off as chance. The multiple recall studies must be regarded as providing at least weak support for the importance of temporal delay in STM.

\section{Probe Recall}

The most elegant previous studies on the effects of rate of presentation on STM are the "probe recall" studies of Waugh and Norman (1965) and Norman (1966). Both studies presented a list of 15 digits followed by a probe digit that had appeared earlier in the list. Subjects were to recall the digit that had followed the probe digit earlier in the list. To a first approximation, both studies found rate of decay to be a function of the number of intervening items, irrespective of rate of presentation. However, there are some trends in these data that suggest that temporal delay may also be important.

The Waugh and Norman study used auditory presentations and two rates, $1 / \mathrm{sec}$ and $4 / \mathrm{sec}$. The plot of probability of correct recall against number of intervening items clearly shows that the $4 / \mathrm{sec}$ curve is below the $1 / \mathrm{sec}$ curve for short delays (few intervening items), but systematically above it for long delays (many intervening items). This suggests that $4 / \mathrm{sec}$ items were less well learned in STM, but decayed at a slower rate, as measured in items, than the $1 / \mathrm{sec}$ items.

The Norman study used both auditory and visual presentation with rates of $1 / \mathrm{sec}$, $4 / \mathrm{sec}, 7 / \mathrm{sec}$, and $10 / \mathrm{sec}$. Norman also transformed the probability decay curves to strength decay curves, making two sets of assumptions: (a) Correct and incorrect alternatives have overlapping normal distributions of strength, with all incorrect alternatives having the same strength distributions and the variances of corrcct and incorrect strength distributions being equal. (b) Subjects choose the alternative with the greatest strength (of association to the probe item).

Norman concluded that decay was strictly a function of the number of intervening items, irrespective of rate of presentation, that is, that rate of decay was a constant, 
when measured as a function of number of intervening items rather than time. However, once again, decay rates, measured in items, do tend to decrease with increasing rate of presentation, suggesting that temporal delay may also be important. Furthermore, a number of other features of Norman's study make the results inconclusive with regard to the presence or absence of temporal decay in verbal STM.

First, $7 / \mathrm{sec}$ and $10 / \mathrm{sec}$ rates seen, introspectively, too fast to permit altending to each item as it is presented. Much of the perception of the items may be done only after the entire list has been presented, possibly using a preperceptual auditory or visual very-short-term memory with rather different properties from the postperceptual STM which handles lists presented at $1 / \mathrm{sec}$ for auditory and visual presentation and $4 / \mathrm{sec}$ for auditory presentation. Visual lists successively presented at rates of $0.75 \mathrm{sec} /$ item or slower are stored largely in a phonetically coded memory (Conrad, 1964). Visual presentation at $4 / \mathrm{sec}$ also seems to be a little too fast for successive attention to each item and transformation into the phonetically coded STM.

If one is concerned about these introspective differences then one should focus on the $1 / \mathrm{sec}$ and $4 / \mathrm{sec}$ auditory decay curves as the only comparison which is virtually certain to be unconfounded. Here, the decay rates determined by Norman are almost exactly half way between what would be expected if decay were strictly a function of the number of intervening items and what would be expected if decay were strictly a function of time.

Second, without questioning the primacy of the number of intervening items for the forgetting curve for STM recall, there is reason to think that recall may be much inferior to recognition as a test of the underlying STM decay process. The reason for this is that a "yes-no" recognition test is "independent from irrelevant strengths" (Bower and Bostrom, 1968; Wickelgren, 1967), which means that, under appropriate conditions, a "yes-no" recognition judgment is based only on the strength of the test item (or test association) in STM, whereas a recall choice necessarily must take many traces into account.

Besides being a more complicated retrieval process to analyze in general, recall tests may introduce a confounding factor into rate of presentation studies, namely, the strength of direct and remote associations. The number of intervening items may be so important in a recall test simply because each intervening item produces a competing remote association to the probe item. Besides being a reasonable possibility, Norman's error analysis indicates that this is occurring in his experiment. Not only may this retrieval interference be emphasizing the importance of the number of intervening items, but also it may be doing so differentially at different rates of presentation, in a manner that further hides the effect of temporal delay. If the relative strengths of direct and remote associations depend in part on the time between the items as well as the number of intervening items, then faster rates of presentation will tend to produce stronger (incorrect) remote associations than slower rates of presentation.

These possible complications in interpreting the results of Norman (1966), the 
slight trends suggesting an effect of temporal delay in both Norman (1966) and Waugh and Norman (1965), and the evidence for an effect of temporal delay in the multiple recall studies indicated that a further study would be helpful in determining the relative roles of time and number of intervening items in the decay of the verbal STM trace. For the reasons mentioned earlier, a probe recognition method seemed most appropriate for such a study.

\section{Strength Theory}

An "atheoretical" analysis of the rate of forgetting in terms of response probability as a function of time or the number of intervening items would simply show the rate of forgetting in STM to be a function of (a) the type of test (recognition, multiplechoice, or recall), (b) the number and nature of the alternatives in multiple-choice or recall, (c) response bias to say "yes" in recognition (measuring response bias by the false recognition rate), with response bias varying greatly over subjects and conditions, (d) the delay itself, whether measured in time or intervening items, and countless other factors. Probability forgetting rates are chaos, whether measured in decrements of probability per second or per item. The question of item decay or temporal decay hardly matters in the face of such variability.

Fortunately, there is a theory of STM which characterizes the memory trace in a manner that yields decay rates which are invariant under enough different experimental manipulations to make the question of temporal vs. item decay both meaningful and answerable. Thus, the present theory will analyze decay rate within the context of a particular theory, namely, strength theory (see Wickelgren, 1969 and 1970, and Wickelgren and Norman, 1966, for the most complete development of the theory). A brief description of strength theory for item recognition memory (where items are presented at a constant rate) is as follows:

The state of an item in STM is characterized by a single real-valued strength, $s$, which starts off at zero, is boosted to $\alpha_{r}$ (which may be a function of rate) upon presentation, and decays exponentially to zero as a function of time or interference or both. In retrieval, only the strength of the test item is considered, and the "yes-no" decision is made by comparing that strength to a criterion, $c$. There is noise in the initial strengths and in the acquisition and retrieval processes (including the criterion). All these sources of noise can be handled by the addition to $s-c$ of a random variable, $X$, which is assumed to be normally distributed with zero mean and a standard deviation which may be a function of the test item. The noise in the decay (storagc) process is assumed to be negligible when rehearsal is carefully controlled. The theory specifies that strength is measurable on an interval scale with the strength of a new item taken as the zero and the noise associated with judgment of a new item taken as the unit. This theory can be represented axiomatically as follows: 
D1. Let $t_{0}=1 / r$ be the time between the onsets of adjacent items, where $r$ is the rate of presentation. Let $t$ be the time since the onset of the last item, $0 \leqslant t \leqslant t_{0}$.

D2. Let $T=(i / r$ or $(i / r)+t)$ be the time delay between presentation and test of an item.

D3. Let $s(T, r)=s\left(i t_{0}, r\right)=s(i, r)$ be the strength in STM of an old test item (presented previously) with $T$ sec. intervening between presentation and test and the rate of presenting new items to be acquired in STM being $r$ items $/$ sec. Let $s\left({ }^{*}, r\right)$ be the strength in STM of a new item (test item not presented previously in the list) tested after a list presented at rate $r$.

A1. (Independence from Irrelevant Strengths in Recognition).

Only the strength of the test item $s(U, r)$ is judged in "yes-no" recognition memory, $U=T, i$, or *.

A2. (Criterion-Decision Rule) Respond "yes" iff $s(U, r)-c+X \geqslant 0$ where $X \sim N[0, \sigma(U, r)], \sigma\left({ }^{*}, r\right)=1$.

A3. (Initial Strength) $s\left({ }^{*}, r\right)=0$.

A4. (Acquisition) $s(0, r)=\alpha_{r}$.

A5a. (Time Decay) $(d s / d T)=-\beta s$.

or

A5b. (Item Decay) $s(i, r)=e^{-\beta} s(i-1, r)$.

or

A5c. (Item-Time Decay) $(d s / d T)=-\beta(t) s$,

$$
\text { where } \beta(t)= \begin{cases}\beta & \text { for } \quad 0 \leqslant t \leqslant t \\ \gamma & \text { for } t_{1}<t \leqslant t_{0}\end{cases}
$$

$\gamma<\beta$.

D4. Let $d^{\prime}(U, r)=\left[s(U, r)-s\left({ }^{*}, r\right)\right] / \sigma\left({ }^{*}, r\right)$.

The item-time decay hypothesis derives from the notion that there is an active STM trace maintenance process, which partially counteracts a passive temporal decay process. This active STM trace-maintenance process interacts with the STM traceacquisition process such that the ability to maintain previous STM traces is less during a period of time (on the order of $0.25 \mathrm{sec}$ ) which is necessary for the perception and acquisition in STM of a new item. During this period the decay rate for previous items is $\beta$. After this period and prior to presentation of the next item to be acquired, the decay rate for previous items is $\gamma$, where $\gamma<\beta$.

From the above theory, it can be shown that the $x$-intercept ( $d^{\prime}$ value in signal detection theory) of the (rating) operating characteristic on a normal-normal probability plot of the data for an old item condition vs. the data for the new item condition 
provides an empirical estimate of $d^{\prime}(U, r)=s(U, r)$. Proofs of this (and formally equivalent matters in signal detection) abound. A simple statement of the proof for recognition memory can be found in Wickelgren and Norman (1966). Rating operating characteristics are explained by Egan (1958), Egan and Clarke (1966), and Green and Swets (1966). Intuitively, the point is that the response probability data for each condition can be converted to empirical estimates of trace strengths for comparison to the predictions of strength theory with each of the three decay assumptions.

The predicted (exponential) decay curves for time decay are given by Eq. 1. Such decay curves for all rates would be parallel straight lines with slopes of $-\beta$ when $\log$ strength is plotted against time, $T$.

$$
s(T, r)=\alpha_{r} e^{-\beta T} .
$$

The predicted (exponential) decay curves for item decay are given by Eq. 2. Such decay curves for all rates would be parallel straight lines of slope $-\beta$ when log strength is plotted against number of intervening items, $i$.

$$
s(i, r)=\alpha_{r} e^{-\beta i} .
$$

If we assume that $t_{1}$ (the time during which trace maintenance is interfered with by acquisition) is approximately constant for all rates such that $1 / r=t_{0}>t_{1}$, then the predicted item-time decay curves are given by Eq. 3, where $\varphi=(\beta-\gamma) t_{1}$. Equation 3 is slightly more complicated than Eqs. 1 and 2 . It predicts that decay curves will depend on both the time delay $T$ and the number of intervening items $i=r T$. However, the time decay curve is still exponential in form, with the decay rate being a linear function of the presentation rate.

$$
s(T, r)=\alpha_{r} e^{-(« r+\gamma) T} .
$$

\section{Method}

\section{Experiment $9 L$}

Procedure. On each trial subjects listened to a list of 9 different letters presented at the rate of 2 or 4 letters/sec, followed by a single test letter (probe), followed by a 5 -sec period during which subjects were to decide whether the probe had appeared earlier in the list on that trial, answering "ycs" or "no," and stating their confidence on a scale from 1 (least) to 4 (most). The list for the next trial was presented starting $3 \mathrm{sec}$ after the end of the decision period for the previous trial, a ready signal being used to terminate the decision period and signal the start of the next trial. All trials were recorded on tape. Sessions lasted about 1 hour. Letters were selected from the following population of consonants: BCDFGHJKLMNPQRSTVXZ. Subjects were instructed on numerous occasions to attend to each item, as it came, and not to group items or ever to rehearse (think of) a previous item (nonrehearsal instructions). Subjects were frequently quizzed on their ability to comply with the nonrehearsal instructions, and they reported no difficulties. 
Design. Each of the 9-serial positions for probes in the list was tested once in a block of 14 trials (correct recognition conditions), and probe letters not in the list were presented 5 times: block (false recognition condition). The order was random. There were 14 blocks/set and 3 sets. Each subject took the 3 sets twice each at each rate, after an initial practice session at each rate, which was not counted. Thus, there were 14 sessions for each subject. There was a 5 -min break between blocks 7 and 8 , with 4 practice trials before blocks 1 and 8 . The rate of presentation for each session was randomized in blocks of 2 sessions.

Subjects. The 4 subjects were students recruited by the Psychology Department and paid for their services. They were run singly or in groups, as their schedules permitted.

\section{Experiment $15 L$}

Procedure. The procedure was identical to that for Exp. 9L, except that lists were 15 letters long, the decision period was $4 \mathrm{sec}$, and there were 3 rates of presentation: $1 / \mathrm{sec}, 2 / \mathrm{sec}$, and $4 / \mathrm{sec}$. All 15 letters were different. Nonrehearsal instructions were employed with no difficulties reported.

Design. Each of the 15 serial positions for "correct" probe letters was tested once in a block of 20 trials and "incorrect" probe letters were presented 5 times/block, in random order. There were $10 \mathrm{blocks} / \mathrm{set}$ for the $2 / \mathrm{sec}$ and $4 / \mathrm{sec}$ rates and $7 \mathrm{blocks} / \mathrm{set}$ for the $1 / \mathrm{sec}$ sets. There were 4 sets for the $2 / \mathrm{sec}$ and $4 / \mathrm{sec}$ rates and 5 sets for the $1 / \mathrm{sec}$ rate. Each set was taken twice each by each subject after an initial practice session at each rate. The rate of the sessions was randomized in blocks of 3 sessions with an extra $1 / \mathrm{sec}$ session in the third and fifth session blocks.

Subjects. Three of the subjects from Exp. 9L were run in Exp. 15L 1 year, and then 3 new subjects were run the next year as a part of a long series of experiments with the same subjects. All were recruited and paid, as before.

\section{Experiment $15 L T$}

The experiment was identical to Exp. $15 \mathrm{~L}$ in every way, except that a tone of 400 to $1000 \mathrm{~Hz}$ (randomly selected in $50-\mathrm{Hz}$ steps) was presented along with the list on every trial. Subjects were to recognize each letter as it came (silently) and then attend to the tone. The purpose of the tone was to provide more control of attention and rehearsal, especially at the slow rates, if that was a problem. No subject in this or the other experiments reported difficulties in complying with the nonrehearsal instructions, but it seemed possible that the tone would give the subjects something else to attend to, in addition to the present letter, and thus make it easier not to think of previous letters. Two subjects used in Exps. $9 \mathrm{~L}$ and $15 \mathrm{~L}$, and one new subject, were used in Exp. 15LT.

\section{RESULTS}

\section{Probabilities}

The empirical probabilities of "yes" responses for $i=0, \ldots, 8$ or 14 , and * for each subject for each rate of presentation are presented in Tables 1, 2, and 3 for Exps. 9L, $15 \mathrm{~L}$, and $15 \mathrm{LT}$, respectively. 
TABLE 1

Probability of "Yes" Responses in Exp. 9L

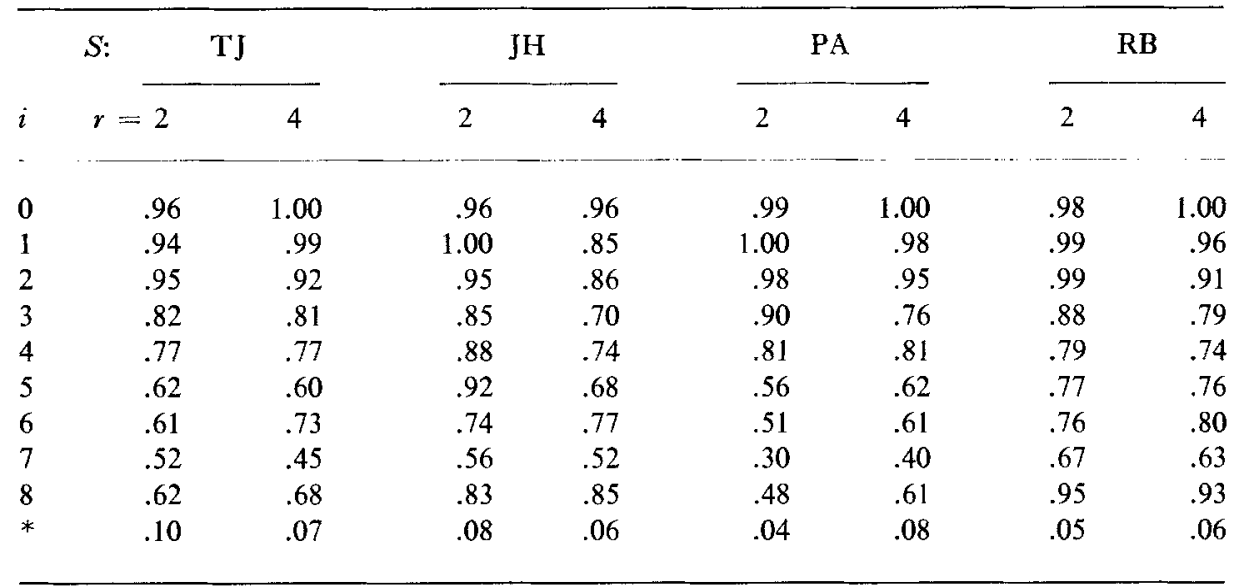

TABLE 2

Probability of "Yes" Responses in Exp. 15L

\begin{tabular}{|c|c|c|c|c|c|c|c|c|c|}
\hline \multirow[t]{2}{*}{$i$} & \multirow[t]{2}{*}{$S:$} & \multicolumn{2}{|c|}{$\mathrm{TJ}$} & \multicolumn{3}{|c|}{$\mathrm{JH}$} & \multicolumn{3}{|c|}{ PA } \\
\hline & & 2 & 4 & 1 & 2 & 4 & 1 & 2 & 4 \\
\hline 1 & .97 & .99 & .99 & .99 & .98 & .91 & .94 & .86 & .94 \\
\hline 1 & 1.00 & .96 & .96 & .99 & .99 & .89 & 1.00 & .90 & .94 \\
\hline 2 & .94 & .95 & .86 & .97 & .92 & .94 & .97 & .87 & .88 \\
\hline 3 & .90 & .91 & .88 & .93 & .91 & .81 & .74 & .83 & .86 \\
\hline 4 & .77 & .78 & .76 & .74 & .74 & .74 & .65 & .62 & .65 \\
\hline 5 & .54 & .63 & .74 & .63 & .72 & .67 & .49 & .43 & .55 \\
\hline 6 & .73 & .72 & .69 & .66 & .68 & .71 & .34 & .43 & .57 \\
\hline 7 & .64 & .45 & .49 & .47 & .72 & .70 & .20 & .37 & .40 \\
\hline 8 & .40 & .49 & .61 & .49 & .58 & .65 & .20 & .26 & .40 \\
\hline 9 & .30 & .38 & .50 & .36 & .30 & .49 & .17 & .23 & .23 \\
\hline 10 & .33 & .26 & .32 & .30 & .41 & .40 & .09 & .16 & .20 \\
\hline 11 & .34 & .39 & .39 & .34 & .26 & .42 & .07 & .18 & .23 \\
\hline 12 & .31 & .25 & .32 & .33 & .40 & .52 & .09 & .14 & .28 \\
\hline 13 & .31 & .22 & .28 & .22 & .24 & .31 & .04 & .07 & .10 \\
\hline 14 & .26 & .26 & .39 & .30 & .35 & .54 & .07 & .17 & .17 \\
\hline$*$ & .18 & .12 & .16 & .17 & .13 & .18 & .09 & .12 & .12 \\
\hline
\end{tabular}


TABLE 2 (continued)

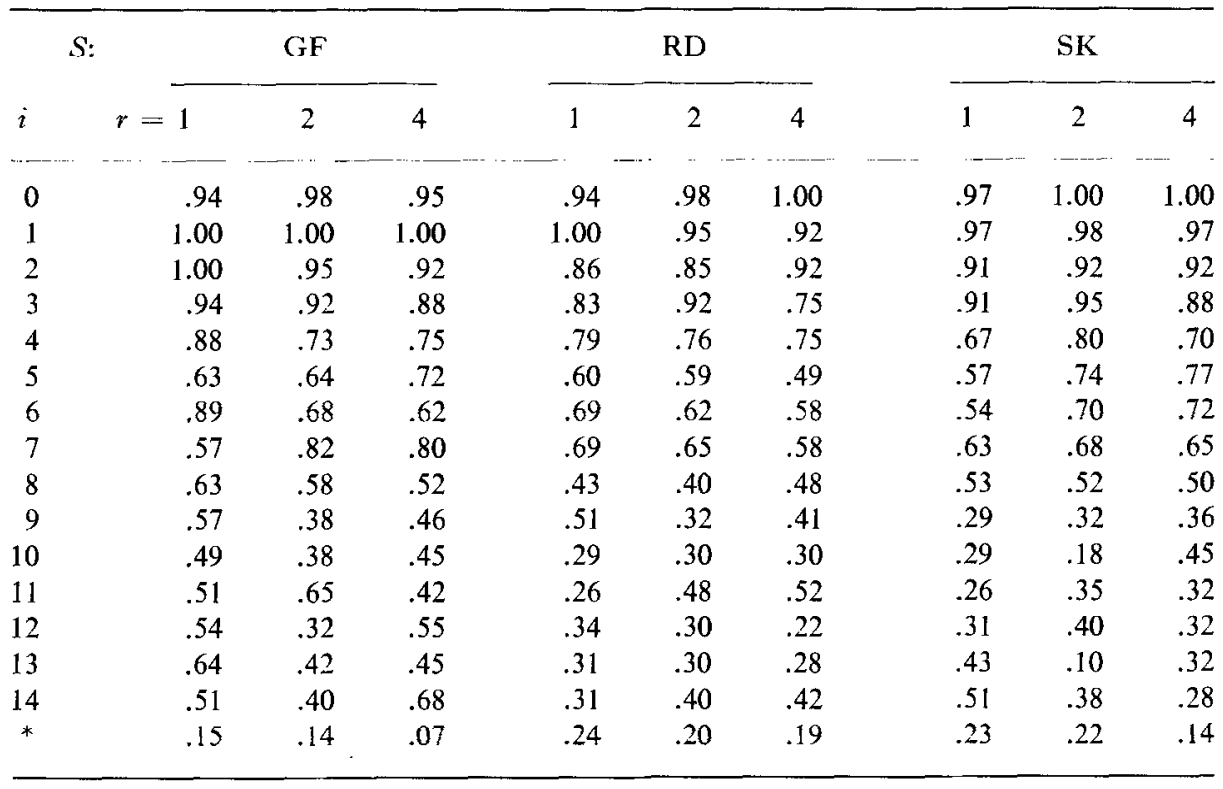

TABLE 3

Probability of "Yes" Responses in Exp. 15LT

\begin{tabular}{|c|c|c|c|c|c|c|c|c|c|}
\hline \multirow[b]{2}{*}{$i$} & $S:$ & \multicolumn{2}{|l|}{$\mathrm{TJ}$} & \multicolumn{3}{|c|}{$\mathrm{JH}$} & \multicolumn{3}{|c|}{ PB } \\
\hline & $r=1$ & 2 & 4 & 1 & 2 & 4 & 1 & 2 & 4 \\
\hline- & & $\cdots$ & & - & & ---- - & & -- & $\cdots$ \\
\hline 0 & .94 & .95 & .98 & .93 & .94 & .92 & .97 & .96 & .99 \\
\hline 1 & .94 & .99 & .88 & .97 & .92 & .83 & .97 & .98 & .95 \\
\hline 2 & .96 & .86 & .84 & .91 & .94 & .82 & .94 & .91 & .87 \\
\hline 3 & .79 & .94 & .88 & .87 & .86 & .80 & .90 & .82 & .74 \\
\hline 4 & .74 & .79 & .76 & .67 & .73 & .78 & .70 & .74 & .73 \\
\hline 5 & .43 & .63 & .61 & .59 & .74 & .56 & .47 & .49 & .55 \\
\hline 6 & .63 & .62 & .59 & .64 & .64 & .79 & .51 & .55 & .44 \\
\hline 7 & .56 & .55 & .48 & .49 & .51 & .52 & .51 & .64 & .63 \\
\hline 8 & .43 & .51 & .51 & .36 & .52 & .56 & .47 & .40 & .49 \\
\hline 9 & .43 & .41 & .55 & .37 & .50 & .42 & .43 & .36 & .36 \\
\hline 10 & .43 & .45 & .36 & .40 & .38 & .42 & .40 & .39 & .40 \\
\hline 11 & .27 & .46 & .42 & .40 & .28 & .36 & .41 & .31 & .32 \\
\hline 12 & .31 & .24 & .35 & .34 & .32 & .34 & .29 & .35 & .46 \\
\hline 13 & .24 & .15 & .20 & .32 & .30 & .31 & .25 & .25 & .38 \\
\hline 14 & .36 & .26 & .37 & .36 & .32 & .66 & .34 & .41 & .40 \\
\hline * & .28 & .20 & .16 & .28 & .21 & .21 & .29 & .26 & .22 \\
\hline
\end{tabular}




\section{Strengths}

Empirical estimates of trace strength in STM were determined for each subject in each delay condition at each rate of presentation. The estimates were obtained in the following way. First, rating operating characteristics for each delay condition $i$ vs. the * condition were plotted by computer on normal-normal probability coordinates. Second, best-fitting straight lines were determined for each operating characteristic using a least squares criterion and ignoring all points which had one or two coordinates beyond \pm 2 standard deviations from the mean. Third, the $x$-intercept of that bestfitting straight line was chosen as the empirical estimate of the strength, $s(i, r)$, of an item tested after $i$ intervening items or equivalently after $i / r$ sec, where items are presented at rate $r$.

Then the logs of the empirical trace strengths for each subject for each rate of presentation for each experiment were plotted as a function of delay, where delay was measured in seconds in one case and in intervening items in the other case. Examples of time decay curves are shown in Fig. 1 and replotted as item decay curves in Fig. 2. With the exception of the first one or two letters in the list, the strength decay curves for each subject in each experiment at each presentation rate did not deviate systematically from a simple exponential decay. That is to say, the strength decay curves did not deviate systematically from linearity on a semilog plot. This replicates for lists of letters the exponential decay of trace strength found in previous probe recognition studies of STM for three-digit numbers (Wickelgren and Norman, 1966) and for the serial order of digit pairs (Wickelgren, 1967).
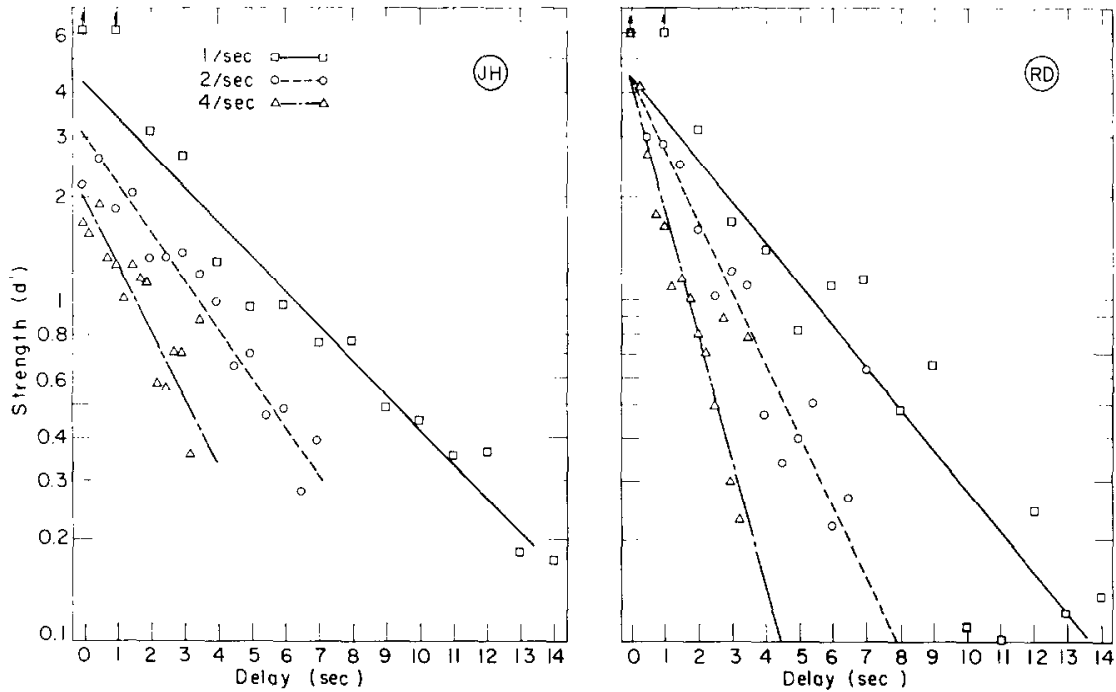

Fic. 1. Semilog plots of strength decay curves for two subjects as a function of time for two subjects with the 15 -letter lists. The straight lines are fit visually. 

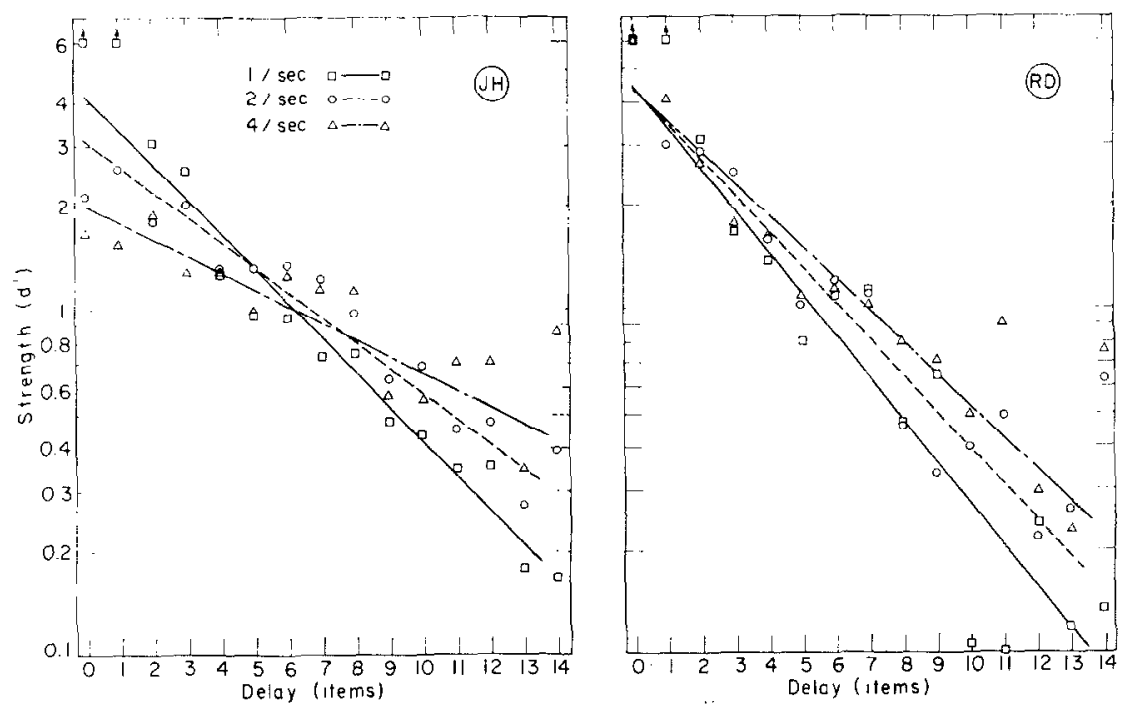

FIG. 2. Semilog plots of strength decay curves for two subjects as a function of the number of intervening items for the 15-letter lists. The straight lines are fit visually.

The first one or two letters in the list were systematically above the best-fitting straight line on a semilog plot presumably because they were better learned (had a higher acquisition parameter), not because they decayed at a different rate or because decay was really not a simple exponential. This interpretation of the very small "primacy" effect usually found in probe recognition memory experiments was validated by the Wickelgren and Norman (1966) study which permitted the decay curve for items from each serial position in a list to be studied independently by using lists of many different lengths. Since, according to the Wickelgren and Norman study, the acquisition parameter and the form and rates of the decay curve were close to identical for all serial positions but the first, it seemed reasonable and efficient to use only one list length and ignore the first item in fitting the strength decay curve. This was what was done and the results showed no systematic deviation from simple exponential decay across all subjects and conditions.

Estimates of the acquisition parameters and decay-rate parameters for each rate for each experiment for each subject were obtained from the zero-delay strength-intercept and slope, respectively, of the best-fitting straight line to the empirical strengths on a semilog plot. This best-fitting straight line was determined visually to be the line which minimized the sum of the absolute deviations of the points from the line, with the following exceptions: First, of course, the point for the first letter in the list (longest delay) was not included. Second, points whose strength values were less than 11 were considered to be anywhere below .1. That is to say, if a theoretical line passed through 
this region, there was no contribution to the total absolute deviation of the theoretical line from the empirical points. Third, in a similar fashion, strengths greater than 4 were considered to be anywhere above 4 . In fitting straight lines to semilog plots of strength decay curves for other cases where there was no systematic deviation from exponential decay, little difference was found between such visually estimated parameters and parameters estimated using a least squares criterion on the empirical strengths or using a minimum chi-square procedure on the "yes-no" probability data (see Wickelgren and Norman, 1966, for a description of these estimation methods applied to probe recognition memory).

According to the item-decay hypothesis, plots of log strength against delay measured in items should be parallel straight lines with the acquisition parameter (zero-delay intercept) being a monotonic nondecreasing function of study time for each item. This was not obtained. Instead, item-decay rate decreased systematically with increasing rate of presentation. If one were to fit the item-decay curves for all three rates of presentation with a single rate parameter and three acquisition parameters, the bestfitting values of the acquisition parameters would frequently not be monotonic nondecreasing with the study time for each item, which is completely contradictory to all studies which manipulate study time independently of the conditions in the delay interval (e.g., Hellyer, 1962; Peterson and Peterson, 1959). Thus, the simple interference position that strengths decay as a function of the number of intervening items is contradicted by the present study.

According to the time-decay hypothesis, plots of log strength against delay measured in seconds should be parallel straight lines with the acquisition parameter being a monotonic nondecreasing function of study time for each item. This was not obtained either. Time-decay rates increased systematically with increasing presentation rate.

According to the itcm-time decay hypothesis, plots of log strength against delay measured in seconds should be straight lines with the acquisition parameter being monotonic nondecreasing with increasing study time (decreasing presentation rate) and the decay rate being a linear function of presentation rate. Values of the acquisition and time-decay rate parameters obtained for the item-time decay hypothesis are shown in Table 4. Decay rates for the same subject are about the same for length-9 and length15 lists, although acquisition is lower in the length-15 lists. The range of individual variation in parameter estimates for the same condition is rather small.

Plots of the time-decay rate against presentation rate for each subject in Exps. 15L and 15LT are shown in Figs. 3 and 4. These figures allow one to assess the adequacy of all three hypotheses. According to the time-decay hypothesis, the plot of time-decay rate against presentation rate should be a horizontal straight line. According to the item-decay hypothesis, time-decay rate should be proportional to presentation rate, that is, a linear function with zero time-decay rate at zero presentation rate. Obviously, neither of these two hypotheses fits the results in Figs. 3 and 4. 
TABLE 4

Acquisition and Decay Parameters for Item-Time Decay

\begin{tabular}{|c|c|c|c|c|c|c|c|}
\hline \multirow[b]{3}{*}{ Exp. } & \multirow[b]{3}{*}{$S$} & \multicolumn{6}{|c|}{ Rate of presentation $(r)$} \\
\hline & & \multicolumn{2}{|c|}{$1 / \sec$} & \multicolumn{2}{|c|}{$2 / \mathrm{sec}$} & \multicolumn{2}{|c|}{$4 / \mathrm{sec}$} \\
\hline & & $\hat{\alpha}_{r}$ & $\hat{\varphi} r+\hat{\gamma}$ & $\hat{\alpha}_{r}$ & $\hat{\varphi} r+\hat{\gamma}$ & $\hat{\alpha}_{r}$ & $\hat{\varphi} r+\hat{\gamma}$ \\
\hline \multirow[t]{4}{*}{$9 \mathrm{~L}$} & $\mathrm{TJ}$ & & & 4.0 & .35 & 4.0 & .48 \\
\hline & $\mathrm{JH}$ & & & 5.7 & .35 & 3.8 & .51 \\
\hline & $\mathrm{PA}$ & & & 10.0 & .60 & 3.9 & .80 \\
\hline & $\mathrm{RB}$ & & & 5.3 & .18 & 4.0 & .43 \\
\hline \multirow[t]{6}{*}{$15 \mathrm{~L}$} & TJ & 4.1 & .21 & 3.5 & .37 & 2.6 & .60 \\
\hline & $\mathrm{JH}$ & 4.2 & .23 & 3.1 & .33 & 2.0 & .46 \\
\hline & PA & 4.8 & .32 & 2.9 & .53 & 2.9 & .82 \\
\hline & GF & 6.4 & .17 & 5.5 & .33 & 5.0 & .53 \\
\hline & RD & 4.5 & .27 & 4.4 & .48 & 4.4 & .85 \\
\hline & $\mathrm{SK}$ & 6.3 & .29 & 5.8 & .55 & 6.3 & .87 \\
\hline \multirow[t]{3}{*}{$15 \mathrm{LT}$} & $\mathrm{TJ}$ & 5.7 & .34 & 5.7 & .51 & 4.1 & .82 \\
\hline & $\mathrm{JH}$ & 4.8 & .32 & 3.6 & .46 & 3.2 & .71 \\
\hline & $\mathrm{PB}$ & 2.8 & .25 & 2.8 & .48 & 2.8 & .82 \\
\hline
\end{tabular}

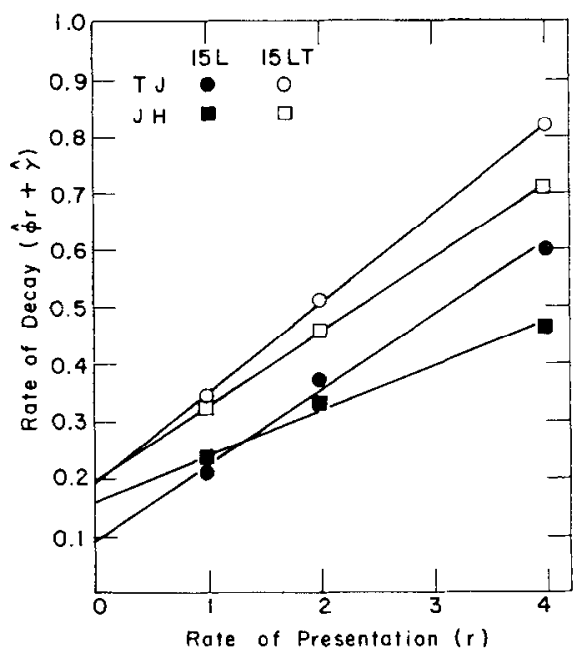

Fig. 3. Time-decay rate as a function of presentation rate for the two $S \mathrm{~s}$ in both Exp. 15L and Exp. 15LT. 


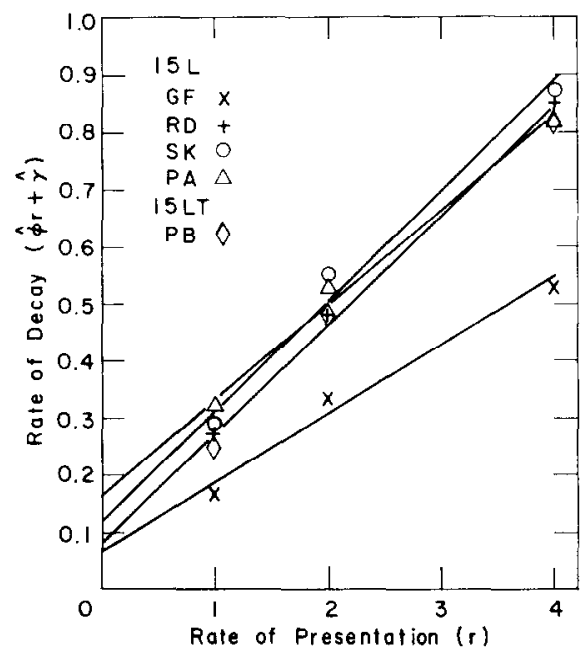

FIG. 4. Time-decay rates as a function of presentation rate for the $S \mathrm{~s}$ who were in only one of Exps. 15L and 15LT.

The item-time-decay hypothesis makes the somewhat weaker prediction that timedecay rate will be a linear function of presentation rate (for rates up to about 4 items/sec with auditory presentation) with a positive time-decay rate at zero presentation rate. This weaker prediction is quite well supported by the results in Figs. 3 and 4. With the dependent variable (decay rate) only varying by a factor of 2 or 3 , the fit of a linear function to only 3 points (which according to any reasonable theory must be monotonic nondecreasing with increasing presentation rate) could hardly be too bad. Nevertheless, making allowances for this, the fit of a linear function seems quite good for all subjects, with a slight, but systematic, deviation from linearity in the direction of less increase in rate of decay with increasing rate of presentation than that predicted by a linear function. This is exactly the kind of systematic deviation that would be produced by a slight violation of the step function assumption for $\beta(t)$ in the direction of a sloping transition from $\beta$ to $\gamma$ or else a slight increase in $t_{1}$ with decreasing rate of presentation. Nothing in the present results suggests that the basic idea of item-time decay is wrong, and actually the step function $\beta(t)$ with constant $t_{1}$ appears to be a remarkably accurate approximation.

Further checks on the item-time-decay hypothesis can be obtained from the present data. In the first place, it is somewhat surprising that the strength decay curves are exponential, given that the decay is a function of both intervening items and time. Item-time decay with step function $\beta(t)$ and constant $t_{1}$ predicts exponential decay, and this certainly must be counted as strong support for the theory.

In the second place, the individual differences in the slopes and intercepts of the 
best-fitting linear functions in Figs. 3 and 4 are within factors of 2 and 5, respectively. Considering that rate of decay can, mathematically, get arbitrarily close to zero or infinity and, psychologically, has a range of around $10^{7}$, comparing the fastest decaying STM and the slowest decaying LTM (see Wickelgren, 1970), factors of 2 and 5 are quite modest for individual differences. This indicates that the good fit of linear functions in Figs. 3 and 4 was not achieved solely by the flexibility afforded by two parameters for three points.

In the third place, the estimated decay rate $(\gamma)$ for $r=0$ is approximately the same as the strength decay rate for a single letter when the delay interval is filled with a task (like backward counting) which controls attention without requiring learning of the material in the delay interval. From other experiments, the fastest such nonlearning, rehearsal-controlling decay rates were established for $\mathrm{RD}, \mathrm{GF}$, and $\mathrm{SK}$, and the agreement was quite close. This comparison is reasonable, if rehearsal was minimal in these tasks and if the reason some other nonlearning, rehearsal-controlling tasks gave slower decay was that they did not control rehearsal as well.

In the fourth place, the effect of the tone in Exp. 15LT is to increase $\gamma$ by comparison to Exp. 15L, for $\mathrm{TJ}$ and $\mathrm{JH}$, the two subjects who where in both experiments. It is not exactly clear what item-time decay should predict to be the effect of adding the additional (tone) stimulus to be attended to. Conceivably, there could have been no effect. However, an increase in $\gamma$ is certainly reasonable, and a decrease in $\gamma$ would have been unreasonable.

\section{Discussion}

The present study demonstrates that both time and the number of intervening items are important factors in producing decay in STM for items. Strictly speaking, this conclusion is valid only for item recognition memory, and it remains to be seen whether the conclusion also holds for order recognition memory (as in Wickelgren, 1967). Very likely, recall involves both item memory and order memory. If this is true, and if recognition and recall use the same memory traces with different retrieval rules, then the present results show that time delay plays a role in short-term recall as well. Furthermore, as was pointed out earlier, the previous multiple and probe recall studies have also suggested that time delay plays some role in producing decay of the STM trace. At the same time, the present study indicates that the most rapid decay of the STM trace occurs as a result of the acquisition of each new item, confirming at least an important component of the Norman (1966) and Waugh and Norman (1965) idea that intervening items produce decay.

The present study also indicates that the decay rate for the STM trace is not a constant, whether measured in time or intervening items. Comparison of the decay curves for probe studies where the intervening material has to be learned and for 
"single-item" studies where the intervening material has to be processed in some way, but not learned, makes the same point. Melton (1963) is right: Decay rate in STM studies is variable.

This fact removes one simple way of demonstrating the existence of separate STM and LTM traces, but, of course, this fact does not prove that the theoretical distinction between STM and LTM or STM and ITM (intermediate-term memory) is vacuous. Nonexponential decay curves that are well fit by the sum of two exponentials with very different time constants (Waugh and Norman, 1965; Wickelgren, 1969) are far more parsimoniously explained by assuming two traces than one trace. More importantly, the neurological studies of patients who have severely impaired ability to form new ITM or LTM traces, but who have normal or almost normal STM traces (Milner, 1966; Scoville and Milner, 1957; Wickelgren, 1968) provide convincing evidence for the existence of two (or more) memory traces. We still do not know how many memory traces there are, but the present study suggests that each memory trace will be characterized by a range of possible decay rates, not by a single decay rate.

\section{REFERENCES}

Aaronson, D. Temporal factors in perception and short-term memory. Psychological Bulletin, $1967,67,130-144$.

Bower, G. H., ANd Bostrom, A. Absence of within-list PI and RI in short-term recognition memory. Psychonomic Science, 1968, 10, 211-212.

Conrad, R. Decay theory of immediate memory. Nature, 1957, 179, 831-32.

ConRaD, R. Acoustic confusions in immediate memory. British Journal of Psychology, 1964, 55, 75-84.

ConRad, R., AND Hille, B. A. The decay theory of immediate memory and paced recall. Canadian Journal of Psychology, 1958, 12, 1-6.

EGAN, J. P. Recognition memory and the operation characteristic. Indiana Univer., Hearing and Communication Lab., AFCRC-TN-58-51, AD-152650, 1958.

Egan, J. P., and Clarke, F. R. Psychophysics and signal detection. In J. B. Sidowski (Ed.), Experimental methods and instrumentation in psychology. New York: McGraw-Hill, 1966.

Fraser, D. C. Decay of immediate memory with age. Nature, 1958, 182, 1163.

GREen, D. M., AND SweTs, J. A. Signal detection theory and psychophysics. New York: Wiley, 1966.

HeLlyer, S. Frequency of stimulus presentation and short-term decrement in recall. Journal of Experimental Psychology, 1962, 64, 650.

Mackworth, J. F. Auditory short-term memory. Canadian Journal of Psychology, 1964, 18, $292-303$.

MeLton, A. W. Implications of short-term memory for a general theory of memory. Journal of Verbal Learning and Verbal Behavior, 1963, 2, 1-21.

Milner, B. Amnesia following operation on the temporal lobes. In C. W. M. WhitTy AND O. L. Zangwill (Eds.), Amnesia. London: Butterworths, 1966.

Norman, D. A. Acquisition and retention in short-term memory. Journal of Experimental Psychology, 1966, 72, 369-381. 
Peterson, L. R., And Peterson, M. J. Short-term retention of individual verbal items. Journal of Experimental Psychology, 1959, 58, 193-198.

Posner, M. I. Immediate memory in sequential tasks. Psychological Bulletin, 1963, 60, 333-349.

Posner, M. 1. Kate of presentation and order of recall in immediate memory. British Journal of Psychology, 1964, 55, 303-306.

Schonfield, D., ANd Donaldson, W. Immediate memory as a function of intraseries variation. Canadian Journal of Psychology, 1966, 20, 218-227.

SCoville, W. B., AND Milner, B. Loss of recent memory after bilateral hippocampal lesions. Journal of Neurology, Neurosurgery and Psychiatry, 1957, 20, 11-21.

Whugh, N. C. AND Norman, D. A. Primary memory. Psychological Reviezv, 1965, 72, 89-104.

WICKELGREN, W. A. Exponential decay and independence from irrelevant associations in shortterm recognition memory for serial order. Journal of Experimental Psychology, 1967, 73, $165-171$.

WiCKELGREN, W. A. Sparing of short-term memory in an amnesic patient: Implications for strength theory of memory. Neuropsychologia, 1968, 6, 235-244.

WICKELGREN, W. A. Associative strength theory of recognition memory for pitch. Journal of Mathematical Psychology, 1969, 6, 13-61.

Wickelgren, W. A. Multitrace strength theory. In D. A. Norman (Ed.), Models of human memory. New York: Academic Press, 1970, in press.

Wickelgren, W. A., and Norman, D. A. Strength models and serial position in short-term recognition memory. Journal of Mathematical Psychology, 1966, 3, 316-347.

ReCeIved: December 9, 1968 